\title{
A GENERALISED EXCHANGE THEOREM FOR MATROID BASES
}

\author{
John Donald and Malcolm Tobey
}

Let $b$ and $c$ be bases of a matroid. Then for any integer $r$, there exists an injection $\sigma$ from $r$-subsets $I$ of $b$ to $r$-subsets $\sigma(I)$ of $c$ such that $b-I+\sigma(I)$ is a base for all $I$. This result has implications for the structure of matroid base graphs.

\section{Generalised base exchange}

Given bases $b$ and $c$ of a matroid $M$, and an element $i \in b$, there always exists an element $j \in c$ such that, with the obvious notational conventions, $b-i+j$ is a base of $M$. This base exchange property of matroids implies stronger exchange properties. For example, Brualdi proved in [1] that there always exists an injection $\sigma$ from $b$ to $c$ such that for all $i$ in $b, b-i+\sigma(i)$ is a base of $M$. In this note we generalise Brualdi's result to arbitrary finite subsets of $b$.

Brualdi's proof depends on Hall's theorem on distinct representatives ([1] or [5, p.505]); indeed, the exchange property appears tailor-made for that theorem. To introduce our ideas we sketch an alternative proof, also depending on Hall's theorem, that avoids using circuits. Hall's theorem states the following: finite sets $X(I)$ have distinct representatives, $I$ ranging over any index set, if and only if the union of any finite number $m$ of the $X(I)$ contains at least $m$ elements.

To use Hall's theorem, first we define our indexed sets. Accordingly, for each $i \in b$ let $X(i)$ denote $\{j \in c \mid b-i+j$ is a base $\}$. The desired injection $\sigma$ from $b$ to $c$ corresponds to a choice of distinct representatives for the $X(i)$. By Hall's theorem, it suffices to show that for any finite $m$ the union $X$ of $m$ distinct $X(i)$ contains at least $m$ elements. Let $I$ denote the $m$ elements of $b$ involved, and for convenience assume $I$ disjoint from $c$. Then there exists, by repeated exchange, a base $b-I+J, J$ a subset of $c-b$ of size $m$. Again by repeated exchange we may delete the elements of $J$ in any order as we move back toward $b$. In particular, for each $j$ in $J$, there exists a base $b-i+j$ for some $i$ in $I$. Since each of these distinct bases is in $X, X$ contains at least $m$ elements.

Through a further application of Hall's theorem, we generalise Brualdi's result to subsets of $b$.

Received 2 March 1990

Copyright Clearance Centre, Inc. Serial-fee code: 0004-9729/91 \$A2.00+0.00. 
TheOREM 1. Let $b$ and $c$ be bases of a matroid $M$ with bases $B$. Then there exists an injection $\sigma$ from $r$-subsets $I$ of $b$ to $r$-subsets $J$ of $c$ such that $b-I+\sigma(I)$ is always a base.

Proof of THEOREM 1: We note that the case $r=1$ is known ([1], or as sketched above) and use induction. Henceforth we assume $r>1$.

For any r-subset $I$ of $b$ let $X(I)$ denote $\{J \mid c \supset J$ and $b-I+J$ in $B\}$. Any set of distinct representatives for the $X(I)$ will define the desired injection, so we try to use Hall's theorem.

Accordingly it suffices to verify the hypothesis of Hall's theorem: given any $m$ $r$-subsets $I_{1}, \ldots, I_{m}$ of $b$, and denoting the union of the associated $X\left(I_{j}\right)$ by $X$, we must have $|X| \geqslant m$. We now get a lower bound on the size of that union in two steps. First we label a bunch of elements of that union, and secondly we show that not too many labels denote the same subset.

Each $I_{j}, j=1, \ldots, m$, contains $r(r-1)$-subsets, which of course may be $(r-1)$ subsets of other $I_{k}, k \neq j$. Let $K_{1}, \ldots, K_{t}$ be a listing, without repetition, of all the $(r-1)$-subsets of all the $I_{j}$, and suppose each $K_{p}$ is contained in $n_{p}$ distinct $I_{j}$. Let, by induction, $\pi$ be a bijection between $(r-1)$-subsets $K$ of $b$ and $\pi(K)$ of $c$ such that $b-K+\pi(K)$ is a base. Then each $b-K_{p}+\pi\left(K_{p}\right)$ is a base. Let $S_{p}$ denote the set of $I_{j}$ containing $K_{p}, p=1, \ldots, t$. By the case $r=1$ applied separately to each of the bases $b-K_{p}+\pi\left(K_{p}\right)$ and $c$, there are $n_{p}$ distinct $y_{j p}$ in $c$ such that for $I_{j}$ in $S_{p}, b-I_{j}+\pi\left(K_{p}\right)+y_{j p}$ is a base. We now have a labelled collection of not necessarily distinct subsets $\pi\left(K_{p}\right)+y_{j p}$ belonging to $X$. The number of such labels is, of course, $\sum n_{p}$. It is also $m r$, because there are $r$ distinct $K_{p}$ contained in each of the $m I_{j}$.

Suppose two labels denote the same set:

$$
\pi\left(K_{p}\right)+y_{j p}=\pi\left(K_{q}\right)+z_{k q}
$$

If $p=q$, then by construction $y_{j p}=z_{k q}$. If $p \neq q$, then by the injective property of $\pi, \pi\left(K_{p}\right) \neq \pi\left(K_{q}\right)$. Consequently $z_{k q}$ is in $\pi\left(K_{p}\right)$, so there are at most $r-1$ choices for $z_{k q}$, whence there are at most $r-1$ choices for $\pi\left(K_{q}\right)+z_{k q}$, again by the injective property of $\pi$. Thus there can be at most $r$ equalities of the form $\left(^{*}\right)$ for fixed $\pi\left(K_{p}\right)+y_{j p}$. Because there are $m r$ such labels, the labels must denote at least $m$ distinct subsets. Thus $|X| \geqslant m$.

Curiously, our proof does not establish the existence of an injection from $b$ to $c$ by which the injection from $r$-subsets to $r$-subsets is induced. Obviously a particular injection of $r$-subsets need not result from an injection of the underlying elements, so we may ask whether there necessarily exists any injection of $r$-subsets induced in this way. The associated underlying injection would have to vary with $r$, as shown by the 
example in [1]. For that example, however, underlying injections exist, separately for each $r$, the only new case being $r=2$.

\section{IMPLICATIONS FOR BASE GRAPHS}

Given a matroid $M$ with collection of finite bases $B$, we say that bases $b$ and $c$ of $B$ are adjacent if they differ by one element; that is, if $|b-c|=|c-b|=1$. Nonadjacent bases are independent. More generally we define the distance $d(b, c)$ between arbitrary bases $b$ and $c$ of $B$ to be the number of elements by which they differ: $d(b, c)=$ $|b-c|=|c-b|$. We denote by $N_{s}(b)$ the set of bases a distance $s$ from the base $b$.

The matroid property imposes a certain combinatorial complexity on the sets $N_{s}(b)$. In particular if $d(b, c)=d$, then we can ask about the joint neighbourhood $N_{r}(b) \cap N_{d-r}(c)$ consisting of certain bases between $b$ and $c$. For example, it is nearly obvious that for $d=2, N_{1}(b) \cap N_{1}(c)$ contains a pair of independent bases. This fact along with other graph-theoretical properties has played a role in various attempts to characterise the adjacency properties of matroid bases $[2,3,4]$. Less obviously, but equivalent to the bijection result of [1], $N_{1}(b) \cap N_{d-1}(c)$ contains a set of $d$ independent bases, for arbitrary finite $d$. Theorem 1 directly yields a further generalisation.

THEOREM 2. Let $M$ be a matroid with bases $b$ and $c$, and suppose the distance $d(b, c)=d$. Then $N_{r}(b) \cap N_{d-r}(c)$ contains a collection of $C(d, r)$ independent bases, where $C(d, r)$ denotes the $r$ th binomial coefficient, " $d$ choose $r$ ".

Proof of Theorem 2: If $I_{1} \neq I_{2}$ are $r$-subsets of $b$ and $\sigma$ is the bijection of Theorem 1, then $\sigma\left(I_{1}\right) \neq \sigma\left(I_{2}\right)$. Thus $b-I_{1}+\sigma\left(I_{1}\right)$ and $b-I_{2}+\sigma\left(I_{2}\right)$ are nonadjacent.

One may neatly visualise the bases $B$ of a matroid $M$ as providing the vertices of a graph $B(M)$, the matroid base graph of $M$, whose adjacencies are the same as the adjacencies of the bases $[2,4]$. From that point of view, a particularly simple matroid results from the $d$-fold product of a 2-base matroid. Its base graph is the $d$-cube, for which Theorem 2 is tight, albeit trivial. At an opposite extreme one has the complete matroid $B_{d, d}$ consisting of all $d$-subsets of a $2 d$-set. For example, in $B_{4,4}$ bases $b$ and $c$ at a distance 4 admit 12 nonadjacent bases in $N_{2}(b) \cap N_{2}(c)$. If the union of shortest paths between bases a distance $d$ apart always contained a $d$-cube, Theorem 2 would be an immediate corollary. Examples like that in [1] show, however, that there need be no such $d$-cube. We have no direct explanation for the presence of so much independence in the joint neighbourhoods of matroid base graphs.

\section{REFERENCES}

[1] R.A. Brualdi, 'Comments on bases in dependence structures', Bull. Austral. Math. Soc. 1 (1969), 161-167. 
[2] C.A. Holzmann, P.G. Norton and M.D. Tobey, 'A graphical representation of matroids', SIAM J. Appl. Math. 25 (1973), 618-627.

[3] J.D. Donald, C.A. Holzmann and M.D. Tobey, 'A characterization of complete matroid base graphs', J. Combin. Theory Series B 22 (1977), 139-158.

[4] S.B. Maurer, 'Matroid base graphs I', J. Combin. Theory Series B 14 (1973), 216-240.

[5] F. Roberts, Applied Combinatorics (Prentice-Hall, Englewood Cliffs, New Jersey, 1984).

Department of Mathematical Sciences

San Diego State University

San Diego, CA 92182

United States of America
Mathematics Department

Southwest State University

Marshall, MN 56258

United States of America 